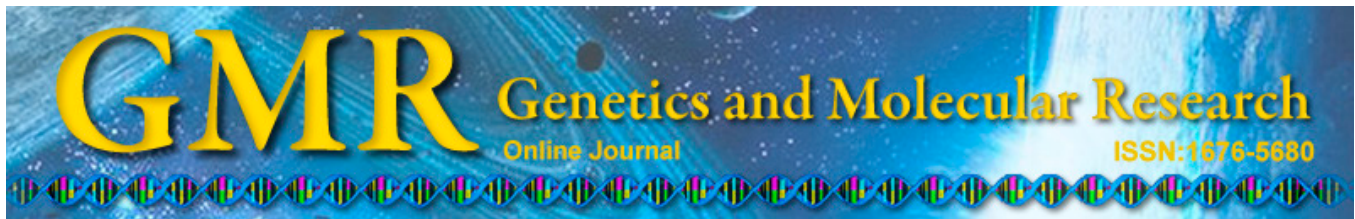

\title{
Infection of Plasmodiophora brassicae in Chinese cabbage
}

\author{
R. Ji*, L. Zhao*, M. Xing, X. Shen, Q. Bi, S. Peng and H. Feng \\ Liaoning Provincial Key Laboratory of Genetics and Breeding of Cruciferous \\ Vegetable Crops, Department of Horticulture, Shenyang Agricultural University, \\ Shenyang, China
}

*Theses authors contributed equally to this study.

Corresponding author: H. Feng

E-mail: fenghuiaaa@263.net

Genet. Mol. Res. 13 (4): 10976-10982 (2014)

Received January 30, 2014

Accepted July 21, 2014

Published December 19, 2014

DOI http://dx.doi.org/10.4238/2014.December.19.20

\begin{abstract}
Brassica crops infected by Plasmodiophora brassicae can produce root galls (clubroots) and be prevented from growing normally. To understand the series of changes that occur in the host root during root gall production, the resistance character of 21 Chinese cabbage lines were identified and then resistant and susceptible lines were used for infection observation. Hydroponic technology system was used for plants growing, and the infection process of $P$. brassicae in the roots of resistant and susceptible Chinese cabbage was examined based on morphology and microscopic characteristics using micoscope. In susceptible Chinese cabbage, the root hair infection stage occurred over approximately 7 days after inoculation, the cortical infection happened over approximatly 14 days after inoculation, and clubroots formed in approximately 30 days after inoculation. However, in resistant Chinese cabbage, the pathogen could be prevented and maintained in the root hair infection stage. This research provides a foundation for the subsequent studies of cabbage resistance of $P$. brassicae.
\end{abstract}

Key words: Chinese cabbage; Hydroponic system; Infection progress; Plasmodiophora brassicae; Resistant lines; Susceptible lines 


\section{INTRODUCTION}

Clubroot of crucifers, caused by the obligate parasite Plasmodiophora brassicae, is characterized by the formation of conspicuous root galls. It was first discovered in Russia in 1878 and named P. brassicae (Voronin, 1878). Clubfoot is a fungal disease present on cultivated and wild cruciferous plants. This disease was identified in the Mediterranean and southern Europe and is now widely distributed worldwide; the problem is particularly serious in temperate zones. Clubfoot recently became common in most provinces and autonomous regions in China (Yang, 1990).

The life cycle of $P$. brassicae consists of 3 phases: survival in the soil, root hair infection, and cortical infection (Kageyama and Asano, 2009), but the effects in Chinese cabbage infected with $P$. brassicae are unclear, including the types of reactions and the lengths of different infection phases. In this study, we examined the morphological and internal changes of Chinese cabbage root during infection by $P$. brassicae. Changes in the root regarding gall development at different stages were examined using light microscopy and histological analysis. Our results may be useful for studies aimed at overcoming clubroot resistance in Chinese cabbage.

\section{MATERIAL AND METHODS}

\section{Plant materials}

The seeds of 21 lines of Chinese cabbage were kindly provided by the Liaoning Provincial Key Laboratory of Genetics and Breeding of Cruciferous Vegetable Crops, Shenyang. These lines are used to carry out resistant studies in the horticulture greenhouse of Shenyang Agriculture University.

\section{Preparation of spore suspension}

Clubroots were collected from the test fields of Shenyang Agriculture University, cleaned in running tap water, and stored at $-20^{\circ} \mathrm{C}$. Resting spores were extracted as described by Castlebury et al. (1994) with some modifications. Mature root galls of Chinese cabbage were homogenized using a juice extractor (Joyoung, Ji'Nan, China). The homogenate was filtered through 8 layers of gauze and the filtrate was clarified by centrifugation 2 times (Biofuge Stratos, Thermo Scientific, Waltham, MA, USA) at $3000 \mathrm{rpm}$ for $9 \mathrm{~min}$ and $4000 \mathrm{rpm}$ for 12 min. The resting spores precipitate was resuspended in sterile water, adjusted to a concentration of $1 \times 10^{7}$ spores $/ \mathrm{mL}$, and stored in $4^{\circ} \mathrm{C}$.

\section{Resistance of different Chinese cabbage lines}

For inoculation with soil bacteria, the clubroots collected from severely disease-affected areas in Shenyang were prepared as spore suspension. The spore suspension was mixed with turf to a final concentration of $1.0 \times 10^{7}$ spores/g turf. The mixture was decayed over 2 days and then used as inoculated soils. Seeds of Chinese cabbage were surface-sterilized with $70 \%$ ethanol for $1 \mathrm{~min}$ and $10 \%$ hydrogen peroxide for $1 \mathrm{~h}$, followed by rinsing 3 times in sterile distilled water (Asano et al., 1999). Surface-sterilized seeds were incubated for 1 day in the dark at $25^{\circ} \mathrm{C}$. The seedlings were planted in the decayed inoculated soil and grown in 
the greenhouse at $20^{\circ}-25^{\circ} \mathrm{C}$. The roots were collected approximately 4 weeks after inoculation and washed thoroughly under running tap water to remove soil particles. Next, the incidence of disease was examined.

\section{Hydroponic growth of Chinese cabbage}

The seeds of resistant and susceptible lines were placed on Petri dish to accelerate germination for 3-4 days in a plant growth chamber (MLR-30H, Sanyo Biomedical, Osaka, Japan) at $25^{\circ} \mathrm{C}$ under a light-dark cycle of $16: 8 \mathrm{~h}$. The germinations were then transferred into the centrifuge tubes containing water and incubated for 4-5 days. When the first euphylla was available, the plants were transferred into Hoagland solution with a circulating system for which the solutions were changed once every 6-7 days to compensate for the nutrient uptake of the plants.

\section{Cultivation and inoculation of plants}

Cultivation conditions and inoculation of plants were performed as follows. For cultivation, the concentration of Hoagland solution, $\mathrm{pH} 6.0$, was used at $1 \mathrm{X}, 1 / 2 \mathrm{X}, 1 / 4 \mathrm{X}$, and $1 / 8 \mathrm{X}$ concentrations. The optimal concentration was identified and used for cultivation. After cultivation for 3 days in a controlled environment $\left(25^{\circ} \mathrm{C}, 16\right.$-h light, $70 \%$ humidity), plants were inoculated by adding $4 \mathrm{~mL}$ resting spore suspension in $1400 \mathrm{~mL}$ cultivate solution to a standard concentration of $10^{7}$ spores $/ \mathrm{mL}$. Control plants were not treated with spores. Plants were harvested at designated times and used for the experiments.

\section{Microscopic observation}

After inoculation, 5 seedlings from each line were randomly selected every $24 \mathrm{~h}$ and cleaned with tap water containing $0.05 \%$ Tween 80 for approximately $20 \mathrm{~min}$. The taproot starting from hypocotyl was cut into $1-\mathrm{cm}$ sections, used to prepare a squash slide, and the hair infection process was observed under a microscope (ECIPSE 80i, Nikon, Tokyo, Japan). Because of the difficulty in preparing the root on the 30th day, a frozen section was selected to prepare the squash slide. The root was cut into $1-\mathrm{cm}$ sections and then embedded with Tissue Freezing Medium (CMI850UV-1-1, Leica, Wetzlar, Germany), cut into 10- $\mu \mathrm{m}$ thin sections, and observed under the microscope.

\section{RESULTS}

\section{Selection of clubroot-resistant and clubroot-susceptible lines of Chinese cabbage}

Resistant lines were identified using inoculated soil in the greenhouse in February 2013. The results showed that infection rates of lines Sy742, Sy179, and Sy 210 were 0\%, and thus were identified as clubroot-resistant lines; the infected rate of line Sy89, Sy112, Sy134, Sy143, Sy149, Sy166, Sy157, Sy191, and Sy174-2 were 100\%, and thus these lines were considered to be clubroot-susceptible; other lines were considered to be disease-tolerant (Table 1). 
Table 1. Disease resistance investigation of different Chinese cabbage lines.

\begin{tabular}{rlccc}
\hline No. & Lines & Infected & Total & Infected rate (\%) \\
\hline 1 & Sy742 & 0 & 19 & 0 \\
2 & Sy179 & 0 & 19 & 0 \\
3 & Sy210 & 0 & 19 & 33.3 \\
4 & Sy100 & 6 & 18 & 44.4 \\
5 & Sy211 & 8 & 18 & 56.5 \\
6 & Sy129 & 13 & 33 & 60.8 \\
7 & Sy145 & 14 & 23 & 69.6 \\
8 & Sy124 & 16 & 23 & 75.0 \\
9 & Sy189 & 15 & 20 & 81.8 \\
10 & Sy092 & 9 & 11 & 88.2 \\
11 & Sy186 & 15 & 17 & 100 \\
12 & Sy209 & 16 & 18 & 100 \\
13 & Sy089 & 14 & 14 & 100 \\
14 & Sy112 & 16 & 16 & 100 \\
15 & Sy134 & 23 & 23 & 100 \\
16 & Sy143 & 19 & 19 & 100 \\
17 & Sy149 & 21 & 21 & 100 \\
18 & Sy157 & 20 & 20 & 100 \\
19 & Sy166 & 14 & 14 & 20 \\
20 & Sy191 & 20 & 20 & \\
21 & Sy742-2 & 20 & &
\end{tabular}

\section{Optimal concentration of Hoagland nutrient solution for Chinese cabbage}

After determining germination and seedling survival in distilled water, Chinese cabbage seeds were cultivated in Hoagland nutrient solution at different concentrations of 1X, $1 / 2 \mathrm{X}, 1 / 4 \mathrm{X}$, and 1/8X (Figure 1a-d, respectively), while water solution was used as the control (Figure 1e). Plants on the 10th day are shown in Figure 1. All plants cultivated in Hoagland nutrient solution at different concentrations grew better than those in water and showed greater blooming. Therefore, $1 \mathrm{X}$ and $1 / 2 \mathrm{X}$ Hoagland nutrient solution were used to cultivate Chinese cabbage. However, the roots cultivated in $1 \mathrm{X}$ solution turned green (Figure 1a). Thus, the 1/2x Hoagland nutrient solution was the most suitable for Chinese cabbage.
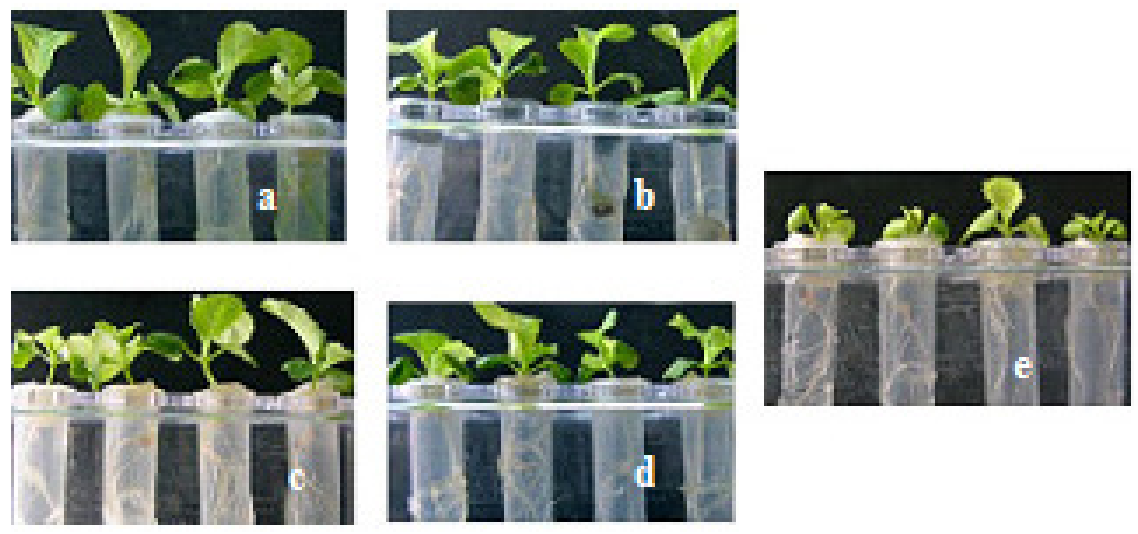

Figure 1. Chinese cabbage of the 10th day cultivated with different concentrations of Hoagland solution. a.-d. The concentrations of Hoagland solution are respectively $1 \mathrm{X}, 1 / 2 \mathrm{X}, 1 / 4 \mathrm{X}$, and 1/8X. e. Water as control. 


\section{Infection of Chinese cabbage with $P$. brassicae}

The progress of root infection and clubroot formation was analyzed by hydroponic inoculation identification combined with microscopic observation. On the 7th day after inoculation, no difference was observed in external morphology among inoculated resistant and susceptible roots and non-inoculated susceptible roots, but microscopic observation revealed that the root hair of susceptible plants had begun to be infected (Figure 2a). On the 14th day after inoculation, no difference was observed in external morphology among the 3 type of roots; however, microscopic observation showed root hair of disease-resistant root in the protoplasm stage. Furthermore, the epidermis protoplasm of disease-susceptible roots had been infected (Figure 2b). On 25-30 days after inoculation, the gall was visible in roots of disease-susceptible lines, and the transverse sections of the roots were broken and were easily sliced, while the roots of non-inoculated and disease-resistant lines were hard and difficult to cut (Figure 2c).

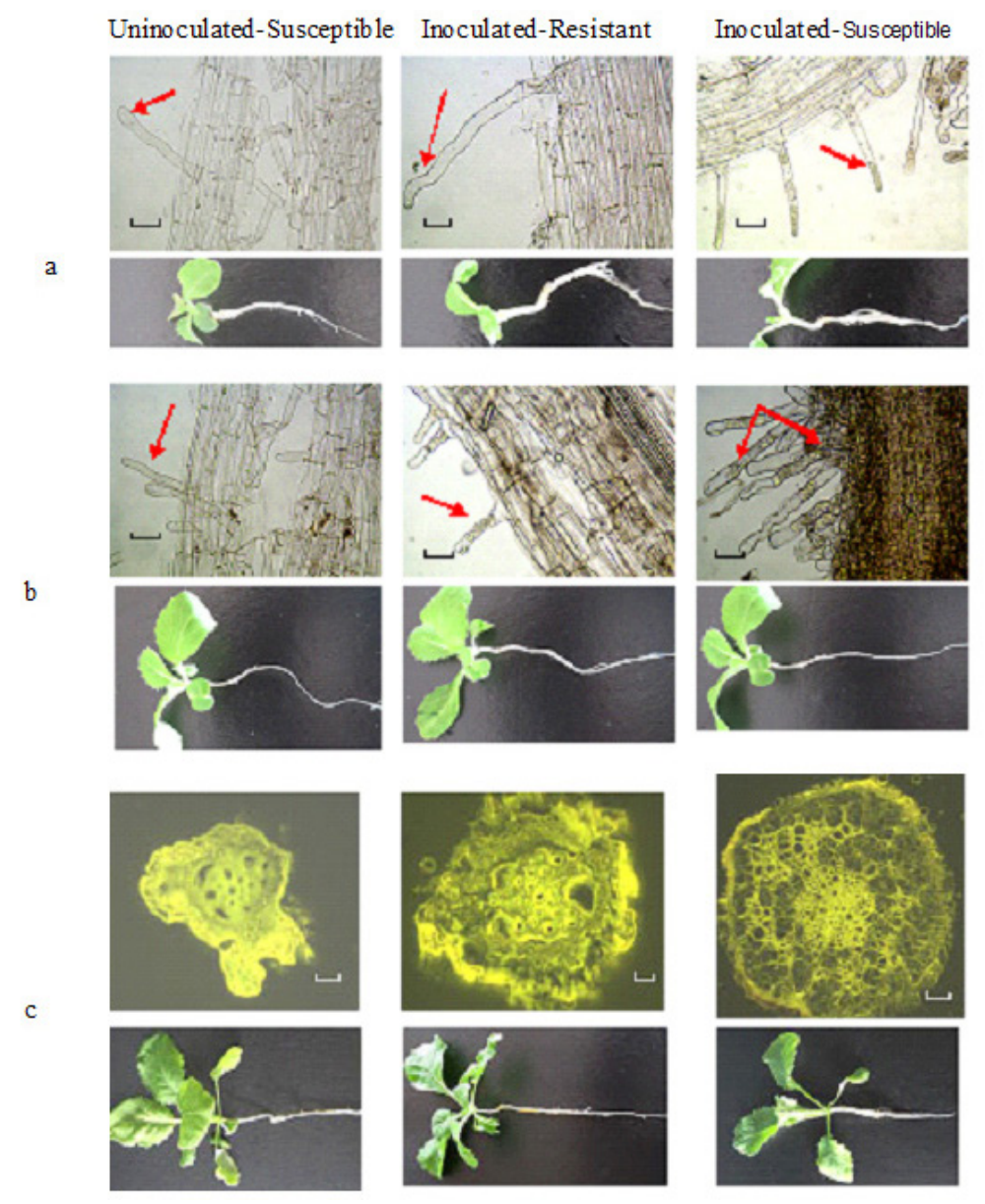

Figure 2. External morphology and microscopic observation of Cabbage roots after inoculating with Plasmodiophora brassicae. a. The 7th day after inoculation. b. The 14th day after inoculation. c. The 30th day after inoculation. Scale bars $=50 \mu \mathrm{m}$. 


\section{DISCUSSION}

Most previous studies of hydroponic nutrient solution used the Hoagland nutrient solution method, organic fertilizer, and the other specifically configured nutrient solutions. Hoagland nutrient solution has been widely applied, but the concentrations used in studies varied according to different plants and cultivation purposes ( $\mathrm{Li}$ et al., 2007; Zhao et al, 2007). In this study, we found that all plants cultivated in Hoagland nutrient solution had bright, dark green leaves, which were superior to control plants (Figure 1a-d). Chinese cabbage cultured in Hoagland solution grew better than in water potentially because it contained relatively complete level of nutrients, allowing the plant growth normally, contributing to plant metabolism, and enhancing photosynthesis (Pan and Dong, 1999). The only study of Chinese cabbage hydroponic technology was reported for chromium stress, in which Hoagland nutrient solution was used (Wang et al., 2013); however, the concentration was not described in detail. By optimizing the nutrient solution concentration, we found that $1 / 2 \mathrm{x}$ Hoagland solution met the demands of Chinese cabbage growth (Figure 1b).

During the life cycle of the P. brassicae, pathogens can live through the winter and summer in soil or animal manure with non-rotted diseased plants. Under appropriate conditions, these resting spores release primary zoospores; when a zoospore swims to the surface of a root hair, it penetrates through the cell wall. This stage is termed the root hair infection stage, and inside root hairs, the pathogen forms primary plasmodia (Agrios, 2005). As reported previously, sporangia are produced 4 days after inoculation (Kageyama and Asano, 2009). In this study, we found that primary plasma inside the root hair of susceptible lines began to multiply, increasing gradually, and more than a quarter of the lines were occupied on the 7th day after inoculation (Figure 2a); Thus, this stage was referred to as the root hair infection stage, similarly to the results of Agrios (2005). Kageyama and Asano (2009) reported that at the root hair infection stage, there were no visible symptoms, which was confirmed in this study (Figure 2b). After secondary zoospores were released, the secondary plasmodia contained 2 nuclei in the early stages of growth, which developed into multinuclear plasmodia during the cortical infection or secondary infection stage (Kageyama and Asano, 2009). We found that 14 days after inoculation, the stage varied gradually from root hair infection to cortex infection, but no external differences were visible (Figure 2b). These plasmodia undergo a series of cell divisions and eventually form multinucleate secondary plasmodia that invade cells in the cortex and stele. Both infected and neighboring uninfected cells undergo extreme hyperplasia (cell division) and hypertrophy (cell expansion), leading to the formation of characteristic galls at 30 days (Figure 2c); these results agree with those of Malinowski et al. (2012) in normal cells, and greater rates of cell division were evident in infected plants on 16, 22, and 26 days after inoculation. Cell division had largely ceased by 32 days after inoculation, while cell division in the hypocotyls of infected plants appeared as a diffuse ring in the vascular cambium (VC) and neighboring progeny cells. Therefore, we clearly determined the life cycle of $P$. brassicae, the dynamic changes of Chinese cabbage roots, and specified the time of each life cycle stage of $P$. brassicae infection in Chinese cabbage. Our results may provide strategies for improving plant tolerance to pathogens. In addition, we found that the disease-resistance preventing pathogen damage mainly occurred during the second stage of cortical infection. 


\section{ACKNOWLEDGMENTS}

Research supported by grants from the National Natural Science Foundation of China (\#312721-57) and the Natural Science Foundation of Liaoning Province (\#2013020071).

\section{REFERENCES}

Agrios GN (2005). Plant Pathology. Academic Press, London.

Asano T, Kageyama K and Hyakumachi M (1999). Surface disinfestation of resting spores of Plasmodiophora brassicae used to infect hairy roots of Brassica spp. Phytopathology 89: 314-319.

Castlebury LA, Maddox JV and Glawe DA (1994). A technique for the extraction and purification of viable Plasmodiophora brassicae resting spores from host root tissue. Mycologia 86: 458-460.

Kageyama K and Asano T (2009). Life cycle of Plasmodiophora brassicae. J. Plant Growth Regul. 28: 203-211.

Li Y, Zhang X, Cai X and Li C (2007). Effect of different nutrient solutions on three foliage-ornamental plants in water culture. J. Anhui Agric. Sci. 23: 7065-7067.

Malinowski R, Smith JA, Fleming AJ, Scholes J, et al. (2012). Gall formation in clubroot-infected Arabidopsis results from an increase in existing meristematic activities of the host but is not essential for the completion of the pathogen life cycle. Plant J. 71: 226-238.

Pan RZ and Dong YD (1998). Plant Physiology, Higher Education Press, Beijing.

Voronin MS (1878). Plasmodiophora brassicae, Urheber der Kohlpflanzen Hernie. Jahrb. Wiss. Bot. 11: 548-574.

Wang WH, Zhong XM, Li BY and Yue ZC (2013). Effects of chromium stress on the growth and chromium accumulation in Chinese cabbage seedlings under solution culture. Acta Agric. Zhejiangensis 25: 492-497.

Yang Y (1990). A preliminary study of fungistatic soil of crucifer clubroot. J. Plant Prot. 17: 127-131.

Zhao L, Mao D, Lin Z, Yang X, et al. (2007). Effects of different nutrient solution on pigment content and photosynthesis of Coleus blumei. Guangdong Agric. Sci. 6: 30-32. 